\title{
Quantifying Sensitivity of Local Site Response Models to Statistical Variations in Soil Properties
}

\author{
José E. Andrade ${ }^{1}$ and Ronaldo I. Borja ${ }^{2}$
}

\begin{abstract}
We perform a combined stochastic-deterministic analysis of local site response using two computer codes, an equivalent linear analysis program SHAKE and a fully nonlinear finite element code SPECTRA. Our goal is to compare the relative sensitivity of the two codes to statistical variations in soil properties. For the case studies, we re-analyze two ground motion records in Lotung, Taiwan, and one ground motion record in Gilroy, California, utilizing the recorded ground motions at the site deterministically as input into the two codes while treating the uncertain soil parameters as random variables. We then obtain empirical cumulative distribution functions of Arias intensity and acceleration spectrum intensity, two measures of cumulative damage, to compare the relative sensitivity of the two codes to variations in model parameters. We show that the two codes exhibit comparable sensitivities to statistical parameter variations, indicating that even in the presence of fluctuations in the soil parameter values it is possible to pursue a fully nonlinear site response analysis with SPECTRA and benefit from its superior accuracy.
\end{abstract}

${ }^{1}$ Graduate Student, Dept. of Civil and Environmental Engineering, Stanford University, Stanford, California 94305-4020, USA. E-mail: jandrade@stanford.edu

${ }^{2}$ Professor, Dept. of Civil and Environmental Engineering, Stanford University, Stanford, California 94305-4020, USA. E-mail: borja@stanford.edu 


\section{Introduction}

Local site response analysis is a critical component of geotechnical earthquake engineering. It facilitates the incorporation of the subsurface material properties as well as surface topography, among others, on the prediction of the amplitude, frequency content, and duration of surface ground motion resulting from a specific input "bedrock excitation.” For horizontal ground surfaces seismic waves are commonly assumed to propagate vertically, and accordingly local site effects are analyzed using vertical soil column models. Among a number of site response analysis codes reported in the literature (Lee and Finn 1991; Li et al. 1992; Pyke 1992; Borja et al. 1999a; Hashash and Park 2002), SHAKE (Schnabel et al. 1972; Idriss and Sun 1992) is by far the most widely used. This code utilizes an algorithm on a one-dimensional soil column model and quantifies local site response based on an equivalent linear analysis procedure. Input soil properties required by the code include the modulus reduction and damping ratio curves, among others. Although these latter curves and other parameters are subject to statistical variations due to the uncertain values of input soil properties, users of SHAKE generally agree that the sensitivity of this code to parameter variations is "acceptable" for most practical engineering applications.

On the other hand, it is generally recognized that fully nonlinear site response models have enormous potential to deliver superior accuracy than equivalent linear models. They are more robust in that they can account for all three components of ground motion in a single analysis. Furthermore, they can handle irregular surface topography and fluid flow effects, as well as capture the generation of excess pore pressures critical for liquefaction analysis. Also, fully nonlinear models can be used to predict finite deformation effects, permanent deformation, softening response, strain localization, and other measures of incremental as well as cumulative damage resulting from an earthquake. However, the price paid by the analyst is increased complexity, number of model parameters, and computational effort (Kramer 1996). It is also believed that fully nonlinear models are likely to be more sensitive to variations in soil properties. Parameter variations could occur not only from laboratory and/or in-situ testing but also from inherent heterogeneity of the soil in the field. Unfortunately, the aspect of model sensitivity to uncertain input soil properties has not been addressed much in the literature. The lack of a clear understanding of this aspect is largely responsible for creating great apprehension on the user's part to pursue fully nonlinear solution analysis for routine applications. 
To understand the sensitivity of a model to uncertain soil parameters, a statistical analysis of the Monte Carlo type is often performed. The procedure is well established and already has been utilized in a number of recent studies (Faccioli 1976; Roblee et al. 1996; Hwang and Huo 1994; Tsai 2000; Andrade et al. 2003; Baturay and Stewart 2003; Bazzurro and Cornell 2004a,b; Borja et al. 2004). First, the soil parameters are treated as random variables with a certain correlation structure that reflects the characteristics of the deterministic model. Then, the deterministic model propagates the uncertainties in the solution by repeated calculations. The result is a band of predictions corresponding to the input range of uncertain soil parameter values. A challenge here lies in the interpretation of an "acceptable" band of predictions (i.e., model sensitivity), since this involves subjective judgment. Note that whereas a narrow band of predictions may imply that the model is quite insensitive to parameter fluctuations, a complete lack of sensitivity is not necessarily the end goal of the exercise since this would mean that regardless of the parameter variations the model predicts one and the same result, and so it is not predictive.

The objective of this paper is to elucidate the sensitivity to parameter variations of a fully nonlinear site response program, called SPECTRA (Borja et al. 1999a; 2000; 2002), relative to that of the program SHAKE. SPECTRA is a nonlinear finite element code that uses a total-stress soil constitutive model based on bounding surface plasticity with a vanishing elastic region (Borja and Amies 1994). This code has been used in the past for local site response and soil-structure interaction analyses (Borja et al. 1999b). Although it exhibits high accuracy, the sensitivity to parameter variations of the program SPECTRA is not well understood. The choice of this code over other fully nonlinear site response codes is motivated by the fact that SPECTRA uses the same parent material parameters as SHAKE, yet it is fully nonlinear. Given that the sensitivity of SHAKE is well understood, the present paper thus offers tremendous opportunity to investigate in great depth the statistical properties of a fully nonlinear site response analysis code against the backdrop of an equivalent linear code.

The general outline of the studies is as follows. First, we employ the standard stochastic-deterministic calculations on both SHAKE and SPECTRA, treating the soil parameters as random variables. Then, using the same input excitation (treated deterministically) and a band of variations for the soil properties, we calculate the empirical cumulative distribution functions (ECDFs) for two damage parameters, herein 
selected as the Arias intensity (Arias 1970) and the Acceleration Spectrum Intensity, or ASI for short (Von Thun et al. 1988), of the calculated motion on the ground surface. The choice of Arias intensity and ASI as measures of cumulative damage is arbitrary; any cumulative measure of damage is acceptable for the analysis at hand. However, scalar damage measures are preferred so that their ECDFs may be clearly presented and compared. To this end we superimpose the ECDFs generated by SHAKE and SPECTRA and compare the performance of the two codes. A direct one-on-one comparison of sensitivities is justified in this case since the two codes receive essentially the same degree of parameter variations. We then repeat the procedure using a narrower band of soil property variations (representing higher certainty in the model parameter values), and again compare the resulting ECDFs. Case studies analyzed include two ground motion records at a downhole array in Lotung, Taiwan, and one ground motion record at a seismically instrumented site in Gilroy, California.

\section{Methodology}

Let $f$ be a response function calculated at any point $\boldsymbol{x}$ and time $t$ in the structure. Symbolically, we can write

$$
f=f(x, t, U, V) .
$$

In the above equation, $U$ is a set of forcing functions and $V$ is a set of parameters used by the "model" (for the present paper, the "model" represents the algorithm used by either SHAKE or SPECTRA). We assume that $U$ is given deterministically whereas $V$ follows a given probability distribution. The objective is to propagate the uncertainties in the set $V$ to the response function $f$. In general, $f$ is not an analytical function, and so we resort to a numerical propagation of uncertainties. To this end we adopt the standard Monte Carlo simulations and obtain empirical cumulative distribution functions (ECDFs) for $f$.

In the present numerical stochastic procedure the elements of $V$ are treated as random variables with a given correlation structure. They are all related to one another via a symmetric correlation matrix given by

$$
\rho_{i j}=\frac{\operatorname{cov}\left(v_{i}, v_{j}\right)}{\sigma_{i} \sigma_{j}},
$$


where $\operatorname{cov}\left(v_{i}, v_{j}\right)$ is the first-order joint central moment (or covariance) of random variables $v_{i}, v_{j} \in V$, representing the linear dependence between two random variables; and $\sigma_{i}$ is the standard deviation of random variable $v_{i}$. Note that $\left|\rho_{i j}\right| \leq 1$, where $\left|\rho_{i j}\right|=1$ signifies a perfectly linear dependence and $\rho_{i j}=0$ implies lack of linear dependence. For the programs SHAKE and SPECTRA, the elements of $V$ are described below.

\section{Random Variables for SHAKE}

SHAKE requires the following soil parameters for input: total mass density $\rho$, elastic shear modulus $G^{\mathrm{e}}$; a plot of modulus reduction $\theta=\bar{G} / G^{\mathrm{e}}$ versus engineering shear strain $\gamma$; and a plot of damping ratio $\xi$ versus $\gamma$. Specification of the spatial variation (in the vertical direction) of these parameters is usually done for improved representation of the soil profile. To account for any significant variation of the parameters in the vertical direction, the soil column model is usually discretized into horizontal layers (Idriss and Seed 1968), and values of the material parameters are prescribed for each layer. The code uses an equivalent linear method for numerical calculations in frequency domain. The uncertainty in the value of $\rho$ at the two sites is too small to be of any significance in the numerical calculations, and so the mass density is assumed to be a deterministic parameter. A "bedrock excitation" is prescribed at the base of the soil column, and the program outputs the ground motion at the surface.

For the analyses conducted with SHAKE, the topology of the matrix $\left[\rho_{i j}\right]$ is as follows. All diagonal components are equal to unity, and all components relating the modulus reduction $\theta_{i}$ with damping ratio $\xi_{i}$ in the $i$ th layer (including the bedrock) are assumed to be equal to -0.75 to account for the strong nearly-linear relationship observed between these two parameters. All other random variables are considered to be mutually independent (i.e., $\rho_{i j}=0$ ).

\section{Random Variables for SPECTRA}

SPECTRA requires the following soil parameters for input: mass density $\rho$; elastic shear modulus $G^{\mathrm{e}}$; radius of the bounding surface $R$; coefficient $h$ and exponent $m$ of the exponential hardening function; and coefficient of proportionality $\chi$ relating the global 
viscous damping matrix $\boldsymbol{D}$ to the global elastic moduli matrix $\boldsymbol{C}^{\mathrm{e}}$, i.e., $\boldsymbol{D}=\chi \boldsymbol{C}^{\mathrm{e}}$. The constitutive model used in this program is a bounding surface elastoplastic solid with a Kelvin viscous enhancement. We refer the readers to Borja and Amies (1994) and Borja et al. (2000) for some general notations and background of the constitutive model. A notable difference between SHAKE and SPECTRA is that whereas the former does not distinguish between viscous and plastic hysteretic damping (it considers both types of damping as viscous), the latter does. Consequently, SHAKE needs the entire spectrum of damping ratio for complete material definition, whereas SPECTRA only needs the asymptotic value of damping ratio at vanishing shear strain to quantify the viscous component of damping.

The radius $R$ of the bounding surface is determined from the equation (Borja et al. 1999a)

$$
R=\sqrt{2} \tau_{\max }, \quad \quad \tau_{\max }=\lim _{\gamma \rightarrow \infty} \theta G^{\mathrm{e}} \gamma
$$

The limiting stress can be alternatively estimated in closed form by assuming a hyperbolic stress-strain relation (Hardin and Drnevich 1972) between the shear stress $\tau$ and shear strain $\gamma$. For a hyperbolic stress-strain curve with an initial tangent modulus $G^{\mathrm{e}}$ and passing through any point $\left(\gamma_{0}, \theta_{0}\right)$, the limiting stress is given by the equation

$$
\tau_{\max }=\frac{\theta_{0}}{1-\theta_{0}} G^{\mathrm{e}} \gamma_{0}
$$

For greater accuracy in estimating $\tau_{\max }$ with equation (4), the point is selected on the "tail" of the modulus reduction curve such that $\theta_{0}$ is as small as possible (or $\gamma_{0}$ is as large as possible). Fig. 1 shows the physical significance of the parameter $R$ as well as illustrates the back-calculation procedure for the limiting stress $\tau_{\max }$. Note that the above hyperbolic approximation for the backbone curve is used only as an alternative approach for estimating $\tau_{\max }$. Once this parameter is prescribed, the bounding surface constitutive model generates its own backbone curve from the exponential hardening law described below. 
The values of the coefficient $h$ and exponent $m$ of the exponential hardening function are obtained from the relationship (Borja and Amies 1994; Borja et al. 2000)

$$
\theta=1-\frac{3}{2 \gamma} \int_{0}^{2 \theta G^{\mathrm{e}} \gamma} Y^{-1} \mathrm{~d} \tau, \quad Y=h\left(\frac{R / \sqrt{2}+\theta G^{\mathrm{e}} \gamma-\tau}{\tau}\right)^{m} .
$$

The above equation reflects the hysteretic damping that occurs in soils subjected to cyclic simple shearing. A standard closed-form procedure for determining the parameters $h$ and $m$ consists of picking two points on the modulus reduction curve, a and b, with coordinates $\left(\gamma_{\mathrm{a}}, \theta_{\mathrm{a}}\right)$ and $\left(\gamma_{\mathrm{b}}, \theta_{\mathrm{b}}\right)$, respectively, and forcing equation (5) to pass through these points (Borja et al. 2000). For greater accuracy, the two points must reflect a significant range of modulus reduction values, e.g., $\theta_{\mathrm{a}}=0.9$ and $\theta_{\mathrm{b}}=0.5$ (see Fig. 1).

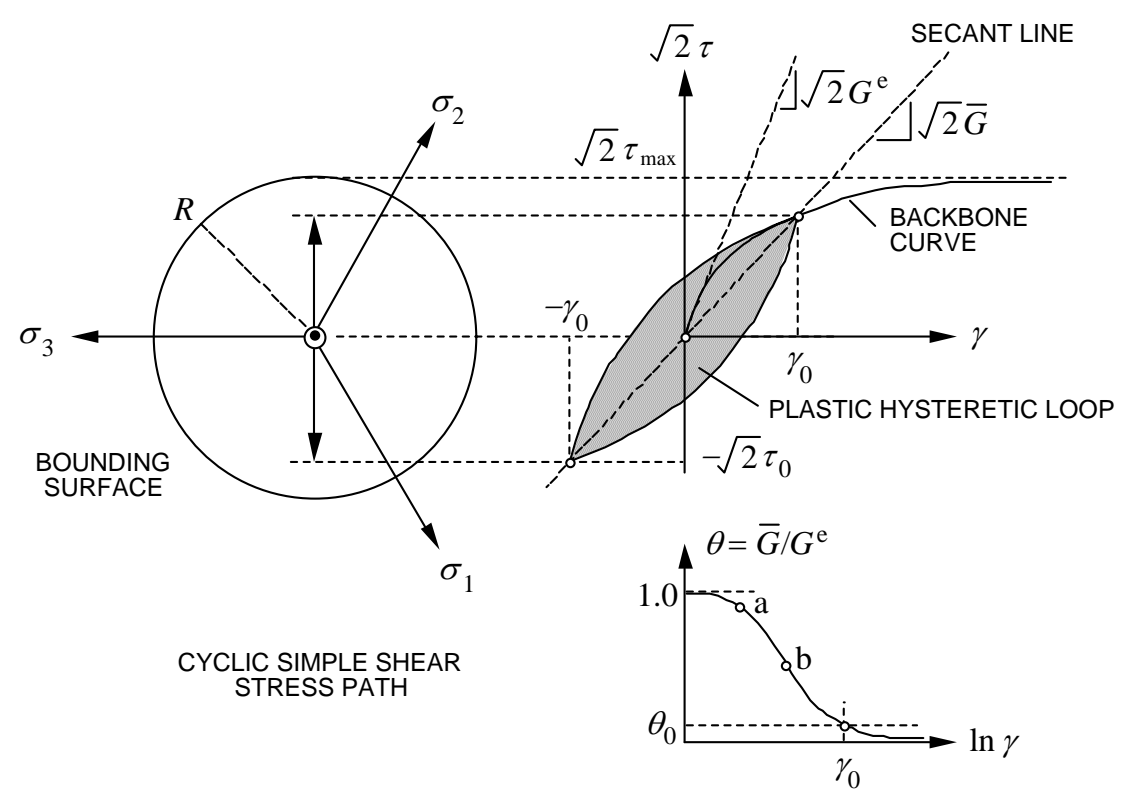

Fig. 1. Deviatoric bounding surface on $\pi$-plane and physical significance of limiting stress $\tau_{\max }$.

Lastly, the value of $\chi$ is determined from the equation (Borja et al. 1999a)

$$
\chi=2 \xi_{0} / \omega
$$


where $\xi_{0}=$ damping ratio at vanishing shear strain, and $\omega=$ angular frequency of the motion. The latter may be taken as the dominant frequency of the input excitation, and is best estimated from the Fourier amplitude spectra of the input motion.

As noted above, SHAKE and SPECTRA are based essentially on the same random variables, and hence, the correlation matrices used by the two codes are practically the same except that SPECTRA does not utilize the random variables for the bedrock. This is because the code treats the input excitation at the soil-bedrock interface as a Dirichlet boundary condition, and so it does not need to know what kind of material is below this interface. Furthermore, since the SPECTRA algorithm automatically calculates the plastic hysteretic damping, it only utilizes the value of damping ratio $\xi_{i}$ at very small shear strain.

\section{Forcing Functions}

Throughout these studies we assume that the forcing functions $U$ in the form of input excitations at the soil-bedrock interface are given deterministically. They consist of three components of motion, north-south (NS), east-west (EW), and up-down (UD). Since SHAKE is an equivalent linear analysis code, input motions are prescribed and analyzed individually for each direction, and the results are combined by superposition (the same modulus and damping ratio profiles have been used for both directions). In contrast, being a fully nonlinear program, SPECTRA analyzes all components of motion simultaneously, although, strictly, the UD motion uncouples naturally from the NS and EW motions because of the deviatoric bounding surface theory used in the code.

Three earthquake accelerograms were used to define the forcing functions in the present study. The first two were obtained from a free-field downhole array in a LargeScale Seismic Test (LSST) site in Lotung, Taiwan, and the third was obtained from a seismically instrumented site in Gilroy, California. For the LSST site, accelerograms from the May 20, 1986 (LSST7, M6.5) and November 14, 1986 (LSST16, M7.0) events were used [see Borja et al. (2002) for a comparison of these two LSST earthquakes]. The intent of analyzing the LSST data from two earthquakes was to see how the amplitude, frequency content, and duration of the earthquakes affect the sensitivity analysis for a given soil site. The third earthquake utilizes the Gilroy 2 ground motions from the October 17, 1989 Loma Prieta earthquake (M6.9). Here, the control motions were taken from the rock site Gilroy 1 located about $2 \mathrm{~km}$ west of Gilroy 2 [see Borja et al. (2000) for further details on the Gilroy earthquake]. The soil at Gilroy 2 is about $170 \mathrm{~m}$ deep, 
much deeper than that at the LSST site, thus allowing the sensitivity analysis to be conducted for a different soil site.

\section{Response Functions}

For the response functions we utilize the calculated Arias intensity (Arias 1970) on the ground surface in the form of ECDF. This response function is given by the integral

$$
I_{\mathrm{a}}=\frac{\pi}{2 \mathrm{~g}} \int_{0}^{t_{0}}[a(t)]^{2} \mathrm{~d} t
$$

where $\mathrm{g}=9.81 \mathrm{~m} / \mathrm{s}^{2}$ and $a(t)$ is the value of acceleration ground response as a function of time. This response function is calculated for both SHAKE and SPECTRA predictions in both EW and NS horizontal directions. Theoretically, $t_{0}=\infty$ determines the total cumulative Arias intensity, but in these studies we select a large but finite value of $t_{0}$ to make the numerical integration feasible. Arias intensity of the recorded ground motions are also calculated and compared with the corresponding ECDFs to gain some insight into the accuracy of the two codes.

We also utilize the Acceleration Spectrum Intensity, or ASI for short (Von Thun et al. 1988), given by the integral

$$
\mathrm{ASI}=\int_{T_{0}}^{T_{1}} S_{\mathrm{a}}(\xi=0.05, T) \mathrm{d} T
$$

ASI represents the area under the acceleration response spectrum curve between periods $T_{0}$ and $T_{1}$ at $5 \%$ damping. Periods of integration are typically from 0.1 to $0.5 \mathrm{~s}$ (see, also, Kramer 1996), although in the present study we take $T_{1}$ to be large enough (20 s) so that the result of the above integral is unambiguous. As mentioned earlier it is possible to choose other measures of cumulative damage, but scalar measures have the advantage in that their ECDFs can easily be presented as two-dimensional plots.

\section{Quantification of Sensitivity}

From the ECDFs of the response functions obtained we quantify the sensitivity to fluctuations in the parameters by using two different measures of dispersion. First, we compare the coefficients of variation (COVs) of the ECDFs obtained using SHAKE and SPECTRA at a given level of dispersion in the parameter set $V$. The COV is a 
dimensionless measure of dispersion and is defined as the ratio between the standard deviation and the mean of a random variable. Second, we define

$$
d=\frac{f(x, t, U, V)-f\left(x, t, U, V_{0.5}\right)}{f\left(x, t, U, V_{0.5}\right)}
$$

as the relative distance from the deterministic response at median parameters $f\left(\boldsymbol{x}, t, U, V_{0.5}\right)$ and calculate the probability of $d$ being within an "acceptable" range, say, $|d| \leq 0.2$ (i.e., $P(|d| \leq 0.2)$ ). We can interpret the value $P(|d| \leq 0.2$ ) as the probability of the deterministic response being within an acceptable range due to parameter fluctuations. The greater the value of $P(|d| \leq 0.2)$, the smaller the sensitivity of the model to randomization of the parameter set $V$. As in the case of the COV, we compare the values of $P(|d| \leq 0.2)$ obtained from the two codes.

\section{Description of Soil Sites}

The local geotechnical profile at the LSST site is composed of a layer of gray silty sand and sandy silt about $20 \mathrm{~m}$ thick, underlain by about $10 \mathrm{~m}$ of gravelly layer resting on a thick deposit of silty clay (Tang 1987). The water table is located approximately at a depth of $1 \mathrm{~m}$ (Anderson and Tang 1989). Unit mass densities of the soil at the LSST site generally vary with depth with the following values (Berger et al. 1989): for 0-15 m, $\rho=1900 \mathrm{~kg} / \mathrm{m}^{3} ; 15-35 \mathrm{~m}, \rho=2040 \mathrm{~kg} / \mathrm{m}^{3}$; and $35-47 \mathrm{~m}, \rho=1940 \mathrm{~kg} / \mathrm{m}^{3}$. The three soil parameters with greatest variability and represented in the sensitivity studies as random variables are $G^{\mathrm{e}}$ and the modulus reduction and damping ratio curves, i.e., $\theta$ versus $\gamma$ and $\xi$ versus $\gamma$, respectively.

Fig. 2 shows the calculated values of the elastic shear modulus $G^{\mathrm{e}}$ as a function of depth from data on shear wave velocities and the measured total unit mass densities at the LSST site. The statistical properties originated primarily from the variability in shear wave velocities, and data points are not represented in Fig. 2 for simplicity in the presentation. The average values of $G^{\mathrm{e}}$ are very similar to those used by Borja and coworkers (1999a;1999b;2000;2002). The calculated mean values vary from a low of 20 $\mathrm{MPa}$ at a depth of $1 \mathrm{~m}$, to a high of $184 \mathrm{MPa}$ at depths 30-35 m; the standard deviations are $9 \mathrm{MPa}$ and $53 \mathrm{MPa}$, respectively. A lognormal distribution is fitted through the data points (not shown) in four of the five layers (bottom layers 2-5) using the method of 
moments. The values of $G^{\mathrm{e}}$ in the upper $17 \mathrm{~m}$ layer (first layer on top) are assumed to follow a linear variation with depth. Assuming a lognormal distribution for $G^{\mathrm{e}}$ at the uppermost $1 \mathrm{~m}$ layer, the corresponding distributions in the lower $16 \mathrm{~m}$ layer are then determined. In addition to fitting the measured values of $G^{\mathrm{e}}$ well, the lognormal distribution has the added convenience of restricting the values of $G^{\mathrm{e}}$ to take only positive values, which is consistent with the physical nature of this particular parameter.

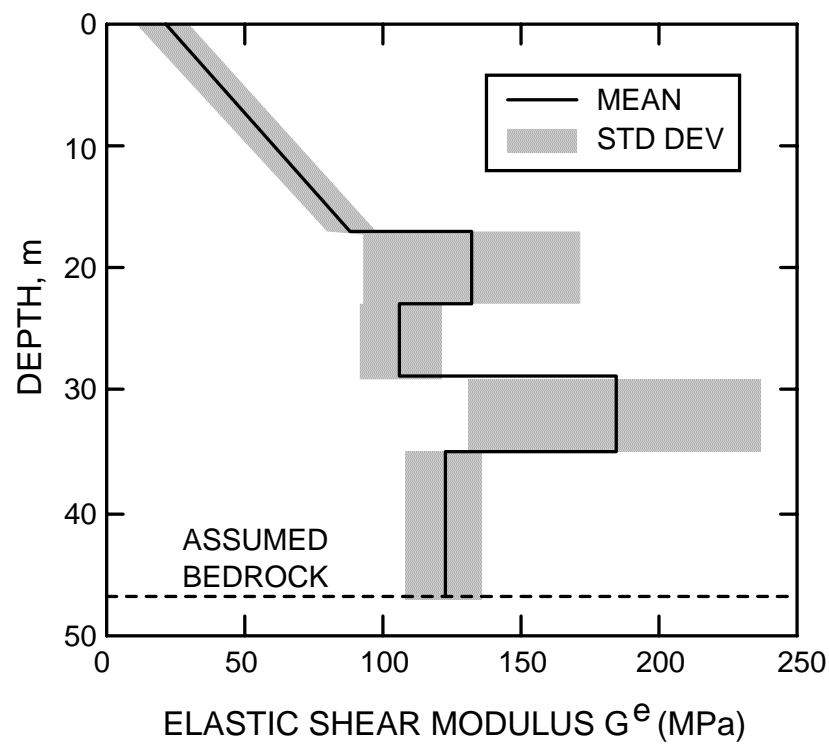

Fig. 2. Elastic shear modulus profile for LSST site in Lotung, Taiwan. Note: boundaries of the shaded region represent one standard deviation away from the mean values.

Elgamal et al. (1995) and Zeghal et al. (1995) reported the values of modulus reduction and damping ratio versus shear strain at different soil depths at the LSST site. Figure 17 of Zeghal et al. (1995) shows the best-fit trends through back-calculations from the three major earthquake events of 1986, and are summarized in Fig. 3 of this paper. Again, for simplicity in the presentation, data points are not represented in Fig. 3. In order to cover the entire $47 \mathrm{~m}$ of soil, the modulus reduction and damping ratio curves obtained at $6 \mathrm{~m}, 11 \mathrm{~m}$, and $17 \mathrm{~m}$ depths are assumed to represent the soil layers at depths 0-6 m, 6-11 m, and 11-17 m, respectively. Additionally, the curves at $17 \mathrm{~m}$ are also assumed to represent the soil at depths 17-47 m. The curves shown in Fig. 3 also contain two gray regions with boundaries representing two standard deviations away from the mean value on each side. The light gray region represents a distribution with a standard deviation equal to SIGMA; the darker region has the standard deviation reduced to SIGMA/2 and represents a distribution with a higher certainty in the soil parameter 
values. The bounds of the light gray region are consistent with those used in Electric Power Research Institute (1993) and encapsulate most of the data points reported by Zeghal et al. (1995). Furthermore, randomization of the curves is accomplished by assuming a normal distribution for both the moduli and damping ratios at a shear strain of 0.025\% and truncating the distributions up and down at two standard deviations as shown in Fig. 3. The remainder of the curve is obtained by linear combinations of the perturbation at $0.025 \%$ shear strain. In these studies we shall use these statistical characterizations to investigate the variations of the ECDFs for Arias intensity as a function of the level of uncertainty in the soil properties.

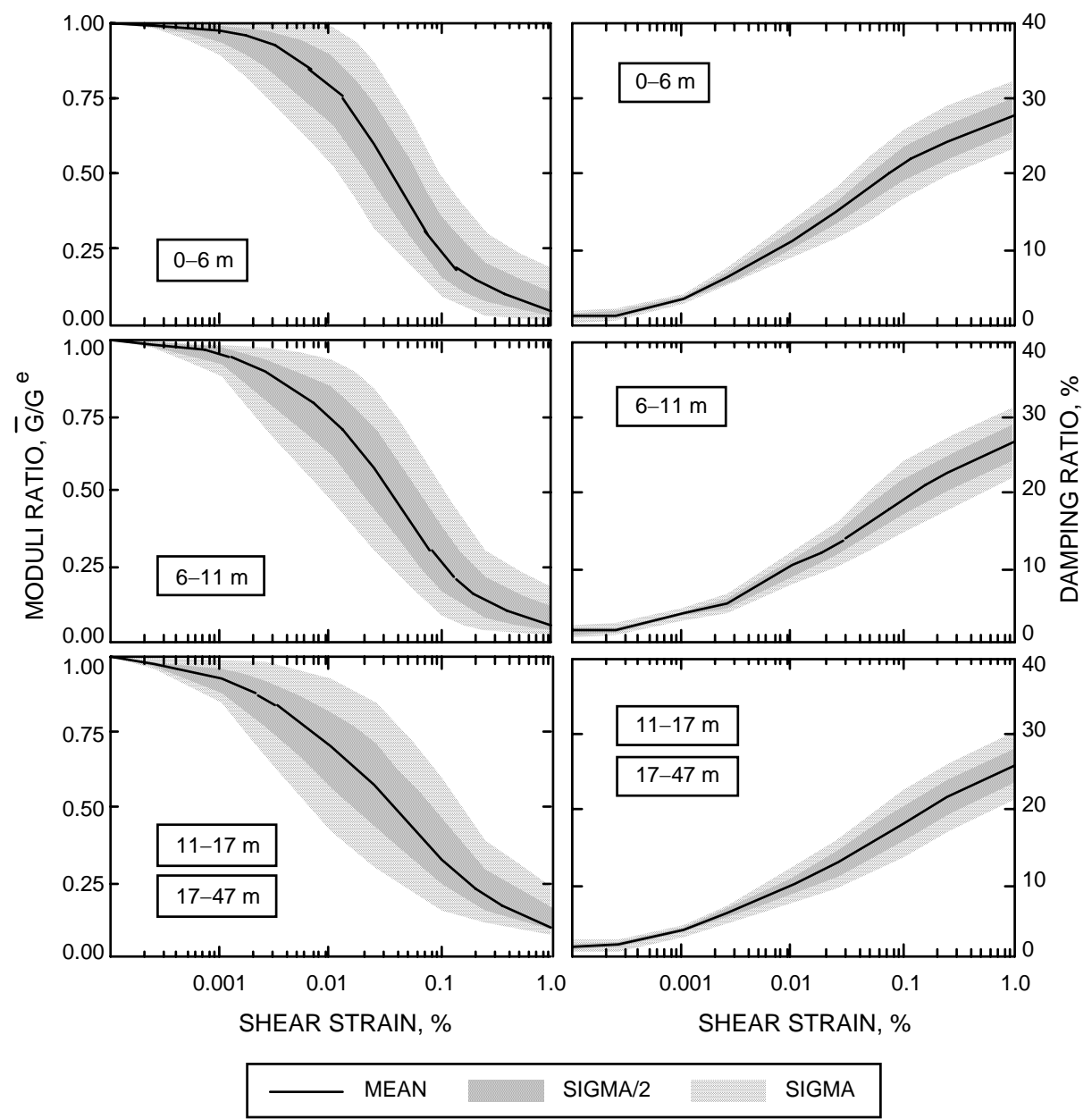

Fig. 3. Modulus reduction and damping ratio curves for LSST site in Lotung, Taiwan.

For the Gilroy case study, the control ground motions from the Loma Prieta earthquake (M6.9, October 17, 1989) were obtained from the rock outcropping site Gilroy 1 located about $2 \mathrm{~km}$ west of the soil site Gilroy 2. The local geotechnical profile 
at Gilroy 2 site consists of sands and clays up to a depth of $40 \mathrm{~m}$. Beyond $40 \mathrm{~m}$ is a deposit of gravel underlain by weathered bedrock at about $170 \mathrm{~m}$ depth. Fig. 4 shows the variation of the mean elastic shear modulus $G^{\mathrm{e}}$ with depth, estimated from results of geophysical testing (Electric Power Research Institute 1993), along with a band of width equal to one standard deviation on each side of the mean value. Similar to the case for the LSST site, the values of the elastic shear modulus are assumed to follow a lognormal distribution with a standard deviation of 0.25 in normal space as in Bazzurro and Cornell (2004a). For the upper $32 \mathrm{~m}$ thick soil layer, the total mass density is on the order of $\rho=1900 \mathrm{~kg} / \mathrm{m}^{3}$; below this depth the total mass density is of the order $\rho=2100 \mathrm{~kg} / \mathrm{m}^{3}$ (Borja et al. 2000).

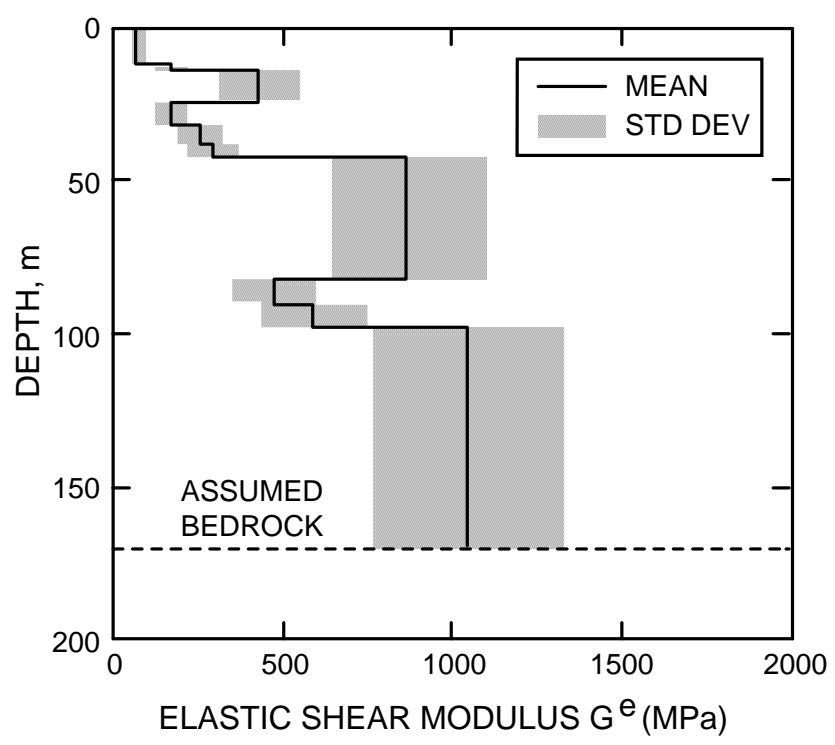

Fig. 4. Elastic shear modulus profile for Gilroy 2 site in California. Note: boundaries of the shaded region represent one standard deviation away from the mean values.

Figure 5 shows the modulus reduction and damping ratio curves for Gilroy 2 site (Electric Power Research Institute 1993). A total of four sets of curves have been reported for this site, representing depths of 0-12 m, 12-24 m, 24-40 m, and depths greater than $40 \mathrm{~m}$. The mean values are superimposed on the two uppermost plots of Fig. 5. Note that the lower modulus reduction curve below $40 \mathrm{~m}$ depth is due to the presence of gravels below this depth. For simplicity in presentation, data points have been omitted from the plots, and only the statistical variations at depth 24-40 $\mathrm{m}$ and depths greater than $40 \mathrm{~m}$ are shown to provide contrast between the highest and lowest moduli ratio values (not shown are the statistical descriptions at depths 0-12 $\mathrm{m}$ and 12-24 m, which are very similar to those at depths $24-40 \mathrm{~m}$ ). The distributions for the moduli and damping ratios 
shown in Fig. 5 are assumed to be Gaussian at a shear strain of $0.025 \%$ and are truncated at twice the standard deviation as shown. The coefficient of variation for these distributions is comparable to those obtained by assuming that the Seed and Idriss (1970) bounds at $0.025 \%$ shear strain are normally distributed about the mean and truncated at twice the standard deviation. Linear combinations of the perturbation at $0.025 \%$ shear strain are performed to obtain the rest of the curve. Comparing the Gilroy 2 and LSST soils, the moduli ratio values for Gilroy 2 soils are generally higher than those for LSST soils, and damping ratio values are accordingly lower. However, the elastic shear moduli are higher at Gilroy 2 site than at the LSST site. These contrasting soil profiles provide a backdrop against which the sensitivities of the two site response codes may be compared.

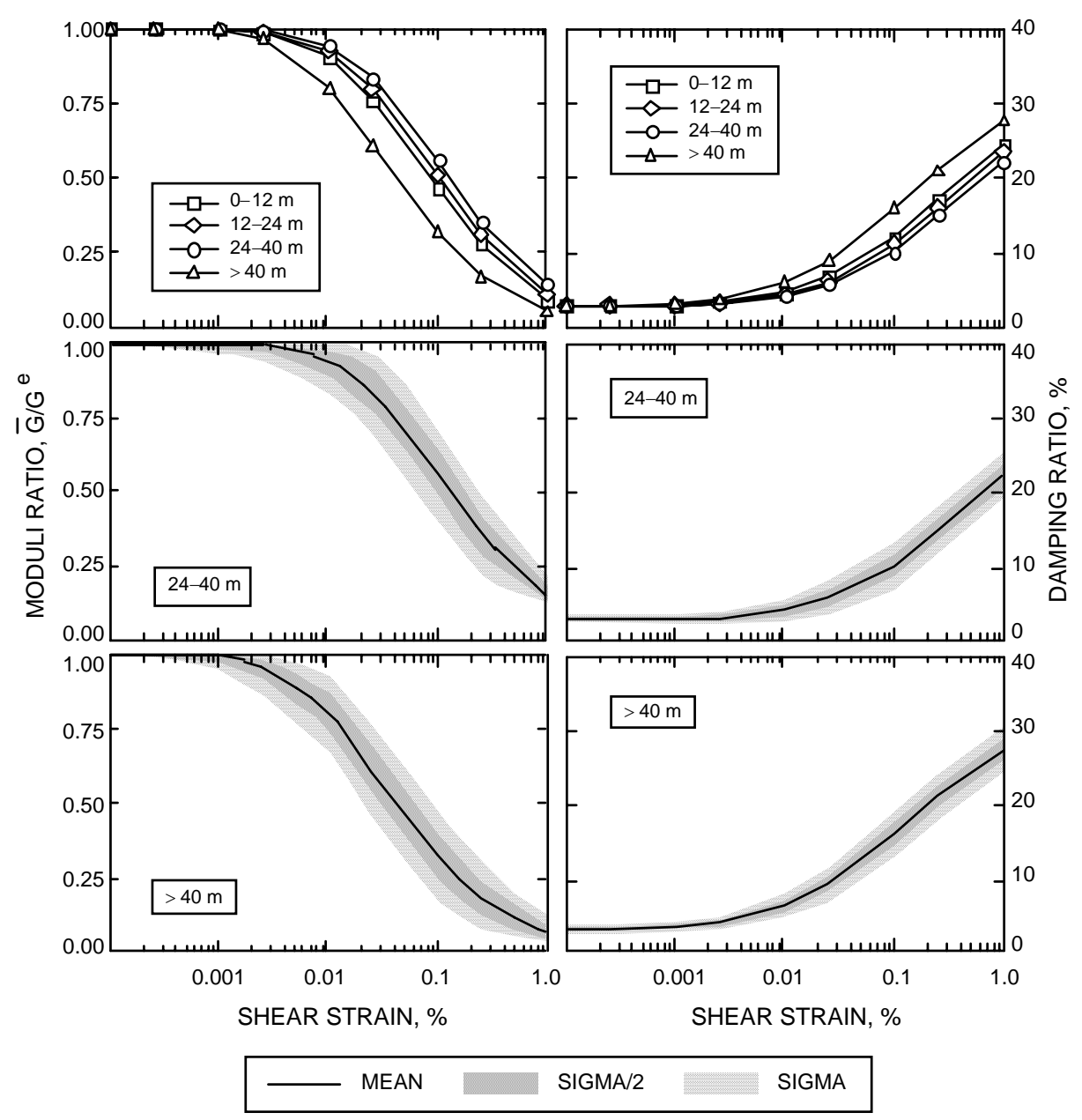

Fig. 5. Modulus reduction and damping ratio curves for Gilroy 2 site in California.

\section{Results and Discussions}


We have coupled the deterministic programs SHAKE and SPECTRA to a structural reliability program called CARDINAL, written by Professor Charles Menun at Stanford University, to perform a series of combined stochastic-deterministic site response analyses. CARDINAL performs four main tasks: (1) generation of random variables; (2) determination of parameters derived from generated random variables; (3) invocation of deterministic programs; and (4) calculation of ECDFs for a given response function. Some numerical integration parameters were held fixed in SPECTRA, including the spatial discretization in the vertical direction (bilinear shape functions) and the Gaussian quadrature rule (standard two-point). Furthermore, we have employed a time-stepping algorithm based on the $\alpha$-method developed by Hilber et al. (1977), with numerical integration parameters $\alpha=-0.1, \beta=0.3025$, and $\gamma=0.6$. These parameters guarantee a second-order accurate, unconditionally stable time-stepping solution capable of introducing some high-frequency numerical damping. Finally, to achieve optimal balance between spatial and temporal discretizations, we have selected a time step $\Delta t$ as close to the critical value as possible ( $\mathrm{Li}$ et al. 2004). For the two Lotung earthquakes where the $47 \mathrm{~m}$ soil column model has been discretized into layers $1 \mathrm{~m}$ thick, we have selected $\Delta t=0.01 \mathrm{~s}$; and for the Gilroy analysis where the $170 \mathrm{~m}$ soil deposit has been discretized into layers $2 \mathrm{~m}$ thick, we have used $\Delta t=0.02 \mathrm{~s}$.

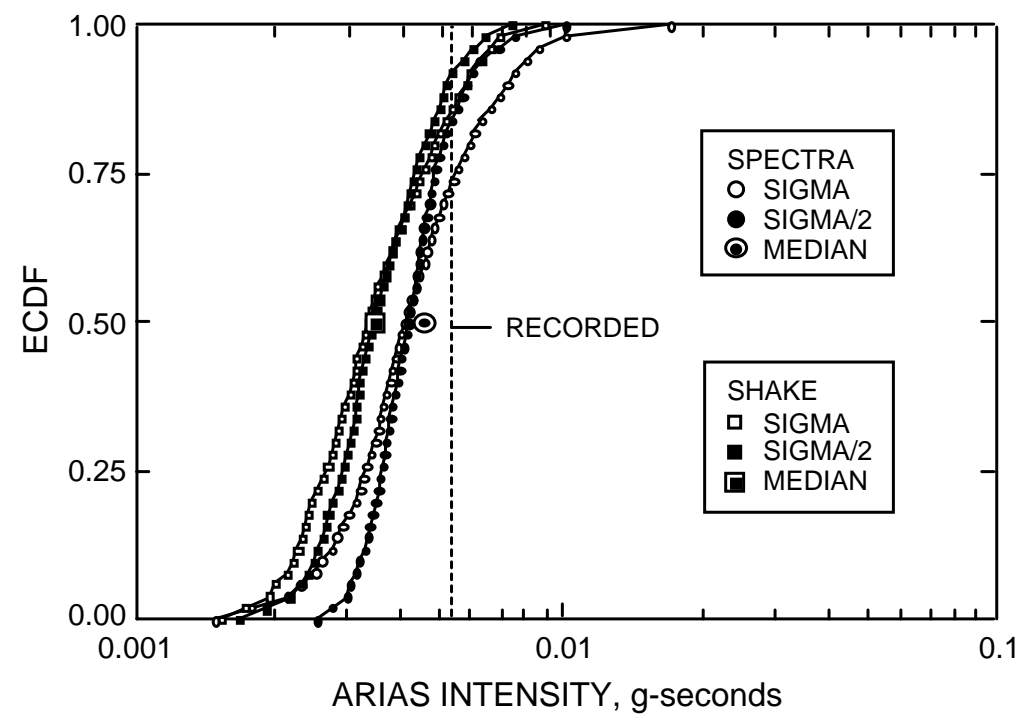

Fig. 6. ECDFs for Arias intensity calculated at $t_{0}=20 \mathrm{~s}$ for LSST7 event using SHAKE and SPECTRA. Solid ticks pertain to distributions obtained using moduli reduction and damping curves with boundaries at two standard deviations and empty ticks represent distributions obtained by reducing the original standard deviations by fifty percent. 


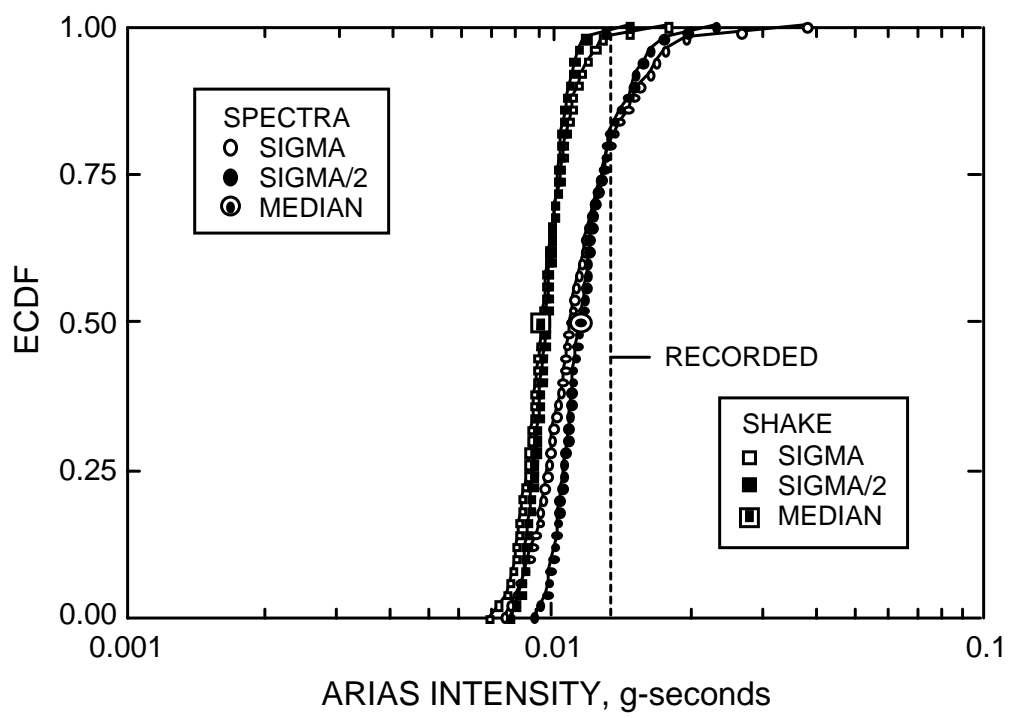

Fig. 7. ECDFs for Arias Intensity calculated at $t_{0}=40 \mathrm{~s}$ for LSST16 event using SHAKE and SPECTRA. Solid ticks pertain to distributions obtained by using moduli reduction and damping curves with boundaries at two standard deviations, and empty ticks represent distributions obtained by reducing the original standard deviations by fifty percent.

The ECDFs of Arias intensity obtained by performing Monte Carlo simulations on both SHAKE and SPECTRA for the LSST7, LSST16, and Gilroy case studies are shown in Figs. 6, 7, and 8, respectively. For each model and seismic event, two ECDFs were generated: one using random input variables at their calculated or assumed mean and standard deviation, and another by reducing the standard deviation of the moduli reduction and damping ratio curves by a factor of 0.5 (labeled in Figs. 6-8 as SIGMA and SIGMA/2, respectively) with the purpose of studying the effect of reducing the level of dispersion or uncertainty in the parameter set $V$. Also shown in the figures are the calculated responses using input parameters at their median values labeled as SHAKE MEDIAN and SPECTRA MEDIAN, i.e., $f\left(x, t, U, V_{0.5}\right)$. These values provide a firstorder approximation to the median value of response function, $f_{0.5}$, and represent "typical” results of performing a deterministic site response analysis for purpose of calculating Arias intensity.

For the LSST7 event the ECDFs of the response function are the results of 500 sample points of Monte Carlo simulations with the input motion applied at $47 \mathrm{~m}$ depth [see Borja et al. (1999a)]. The values of Arias intensity were calculated numerically by integrating over the duration of the strong motion, which is about $20 \mathrm{~s}$ for this event. Fig. 
6 shows the results obtained from SHAKE and SPECTRA using 500 sampling points of Monte Carlo simulations.

\begin{tabular}{|c|c|c|c|c|}
\hline \multirow{4}{*}{ Event } & $\begin{array}{c}\text { Model/Parameter } \\
\text { Dispersion }\end{array}$ & $\begin{array}{c}\text { Mean } \\
\text { (g-s) }\end{array}$ & $\begin{array}{c}\text { Standard } \\
\text { Deviation (g-s) }\end{array}$ & CoV \\
\hline \multirow{4}{*}{$\begin{array}{c}\text { LSST7 } \\
\text { (Fig. 6) }\end{array}$} & SHAKE-SIGMA & $3.78 \mathrm{E}-03$ & $1.37 \mathrm{E}-03$ & 0.36 \\
\cline { 2 - 5 } & SPECTRA-SIGMA & $4.68 \mathrm{E}-03$ & $1.98 \mathrm{E}-03$ & 0.42 \\
\cline { 2 - 5 } & SHAKE-SIGMA/2 & $3.79 \mathrm{E}-03$ & $1.07 \mathrm{E}-03$ & 0.28 \\
\cline { 2 - 5 } & SPECTRA-SIGMA/2 & $4.47 \mathrm{E}-03$ & $1.08 \mathrm{E}-03$ & 0.24 \\
\hline \multirow{4}{*}{$\begin{array}{c}\text { LSST16 } \\
\text { (Fig. 7) }\end{array}$} & SHAKE-SIGMA & $9.68 \mathrm{E}-03$ & $1.42 \mathrm{E}-03$ & 0.15 \\
\cline { 2 - 5 } & SPECTRA-SIGMA & $11.86 \mathrm{E}-03$ & $3.22 \mathrm{E}-03$ & 0.27 \\
\cline { 2 - 5 } & SHAKE-SIGMA/2 & $9.72 \mathrm{E}-03$ & $9.63 \mathrm{E}-04$ & 0.10 \\
\hline \multirow{4}{*}{$\begin{array}{c}\text { Gilroy } \\
\text { (Fig. 8) }\end{array}$} & SPECTRA-SIGMA/2 & $12.15 \mathrm{E}-03$ & $2.26 \mathrm{E}-03$ & 0.19 \\
\cline { 2 - 5 } & SHAKE-SIGMA & $35.78 \mathrm{E}-03$ & $18.88 \mathrm{E}-03$ & 0.53 \\
\cline { 2 - 5 } & SHACTRA-SIGMA & $31.71 \mathrm{E}-03$ & $14.91 \mathrm{E}-03$ & 0.47 \\
\cline { 2 - 5 } & SPECTRA-SIGMA/2 & $29.52 \mathrm{E}-03$ & $12.38 \mathrm{E}-03$ & 0.42 \\
\hline
\end{tabular}

Table 1. Partial descriptors for the ECDFs of Arias intensity obtained from SHAKE and SPECTRA for LSST7, LSST16, and Gilroy events.

From Fig. 6, it can be observed that at each level of dispersion in the set of model parameters $V$ the slope of the ECDFs generated using the two codes are qualitatively very similar. As mentioned above, we can contrast quantitatively the dispersion in the response by looking at the COV for both codes. Table 1 shows three partial descriptors for all the ECDFs obtained at both the LSST and Gilroy sites at both levels of dispersion in the parameter set $V$. For the LSST7 event, we observe that the COV and standard deviation for SHAKE and SPECTRA are about the same.

The ECDFs shown in Fig. 6 can be used to obtain $P(|d| \leq 0.2) \approx 0.41$ for SHAKE and around 0.44 for SPECTRA, at a standard deviation of SIGMA. Similarly, for a standard deviation reduced to SIGMA/2 the probability that $|d| \leq 0.2$ increases to 0.52 for SHAKE and 0.65 for SPECTRA. It is worthy of note that the values of Arias intensity calculated using median soil parameters, $f\left(x, t, U, V_{0.5}\right)$, compare very well with 
the median responses, $f_{0.5}(x, t, U, V)$, from each model, yet the models predict consistently different values of Arias intensity throughout most of the distributions for the LSST7 event. The value of Arias intensity obtained from SPECTRA using median parameters combined with the ECDFs suggests that the deterministic response generated using this code tends to be closer to the recorded value of Arias intensity.

For the LSST16 event the ECDFs of the response function are the results of 500 sample points of Monte Carlo simulations with the input motion applied at $17 \mathrm{~m}$ depth [see also Borja et al. (2002)]. The values of Arias intensity were calculated by integrating numerically the acceleration response history over $40 \mathrm{~s}$, which is roughly the duration of the strong motion for this event. Fig. 7 shows the ECDFs for Arias intensity obtained for SHAKE and SPECTRA. Similar to the LSST7 event, the distributions at a given level of uncertainty in the soil parameters seem to have qualitatively similar spread, with SPECTRA showing a relatively higher sensitivity.

We have also calculated the probability of the deterministic response staying within the established acceptable range, i.e., $|d| \leq 0.2$. At a standard deviation of SIGMA in the input parameters the probability of $|d|$ being less than or equal to 0.2 is around 0.87 for SHAKE and 0.67 for SPECTRA. Similarly, for a reduced standard deviation of SIGMA/2, $P(|d| \leq 0.2)$ is roughly 0.93 and 0.82 for SHAKE and SPECTRA, respectively. Although these results suggest SHAKE exhibits slightly less sensitivity to parameter variation, the value for SPECTRA is not much different. Furthermore, like in the LSST7 event, the deterministic response $f\left(x, t, U, V_{0.5}\right)$ predicted by the nonlinear model is closer to the recorded value as shown in Fig. 7. This result combined with the fact that the resulting ECDFs for this event do not cross at any point suggests that the deterministic predictions obtained by SPECTRA are consistently closer to the recorded value of Arias intensity at $40 \mathrm{~s}$.

Comparing the COVs obtained for the LSST7 and LSST16 events, note the discrepancy in the predicted magnitudes of the COVs particularly at a standard deviation of SIGMA. This can be attributed in part to the fact that there are less random properties in the LSST16 event that can cause dispersion in the response, since for this case we have only modeled a soil column $17 \mathrm{~m}$ deep as opposed to the LSST7 event where we have modeled the entire $47 \mathrm{~m}$ soil column. Additionally, the fact that these results were generated using two different input motions is also expected to contribute to the disparity in the calculated values of COVs. The disparity in the COVs is not a critical issue since, 
as mentioned earlier, we are only concerned here with the relative sensitivity of the codes for a given seismic event.

For the Gilroy event the simulations were produced using 300 sampling points of Monte Carlo simulations. The model is composed of $170 \mathrm{~m}$ of soil column discretized into layers $2 \mathrm{~m}$ thick and analyzed for an input motion having a duration of $20 \mathrm{~s}$. The values of Arias intensity for this event were calculated over 20 s of shifted response at the ground surface. The $0.3 \mathrm{~s}$ shift was introduced to account for the delay in the arrival of the seismic waves traveling from Gilroy 1 to Gilroy 2 sites [see Borja at al. (2000) for further details]. Fig. 8 shows the results obtained from the simulations performed at Gilroy. Note that the distributions at each level of dispersion in the soil parameters have very similar slopes, suggesting similar sensitivities for both SHAKE and SPECTRA. Table 1 shows the COV for the ECDF of Arias intensity obtained from both codes.

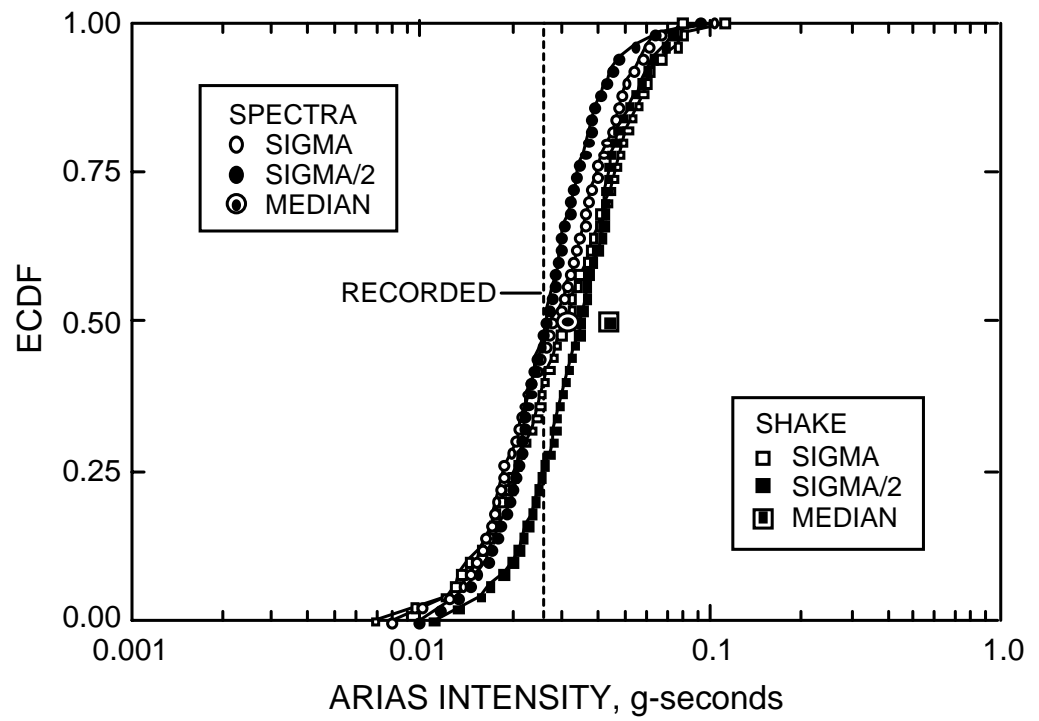

Fig. 8. ECDFs for Arias Intensity calculated at $t_{0}=20 \mathrm{~s}$ for the Gilroy event using SHAKE and SPECTRA. Solid ticks pertain to distributions obtained by using moduli reduction and damping curves with boundaries at two standard deviations, and empty ticks represent distributions obtained by reducing the original standard deviations by fifty percent.

As in the case of the LSST analyses, SPECTRA predicted a deterministic value of the response calculated using median values of soil parameters, $f\left(x, t, U, V_{0.5}\right)$, that is closer to the recorded value of Arias intensity at $20 \mathrm{~s}$. Additionally, if we calculate the probability of the deterministic analysis yielding results within $20 \%$ relative difference 
with the value $f\left(x, t, U, V_{0.5}\right)$ at a standard deviation of SIGMA, we obtain 0.27 and 0.26 for SHAKE and SPECTRA, respectively. Similarly, for a reduced standard deviation of SIGMA/2 the probability for SHAKE increases to 0.42 while that for SPECTRA increases to 0.36. Furthermore, as in both of the LSST events analyzed earlier, the distributions at each level of dispersion in the soil parameters do not cross in general. This suggests that there exists a consistent difference in the response values obtained from the two models, with the deterministic values of Arias intensity obtained from SPECTRA at median input soil parameters being closer to the recorded value of Arias intensity.

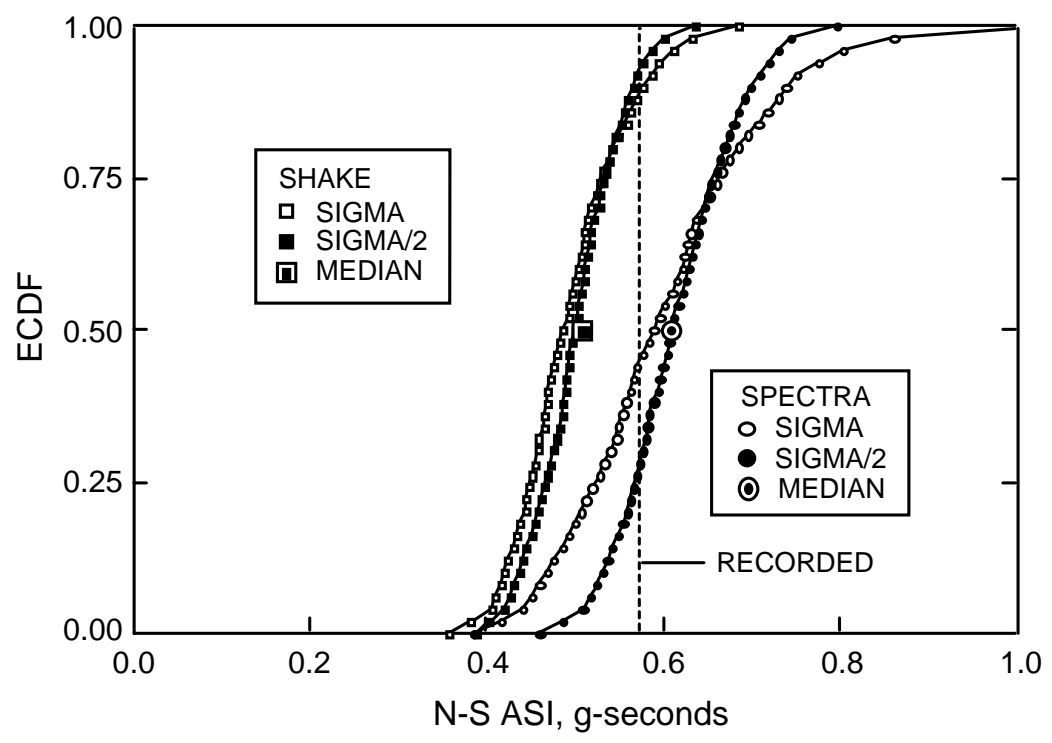

Fig. 9. ECDFs for Acceleration Spectrum Intensity (N-S direction) calculated at $T_{1}=20 \mathrm{~s}$ for LSST7 event using SHAKE and SPECTRA. Solid ticks pertain to distributions obtained by using moduli reduction and damping curves with boundaries at two standard deviations, and empty ticks represent distributions obtained by reducing the original standard deviations by fifty percent.

To demonstrate that other cumulative measures of damage may also be used to compare sensitivities of the two codes, we have also plotted in Figs. 9 and 10 the ECDFs for N-S and E-W ASI generated for the LSST7 event, respectively. The results suggest the same trend, i.e., sensitivities are about the same for the two codes as reflected by the slopes of the ECDF curves being nearly the same, with SPECTRA predicting superior accuracy when median parameter values were used. Furthermore, the ECDFs at each value of dispersion and for each direction do not cross in general. All of these results suggest that the probability of the value of ASI falling within an acceptable range is 
similar for both codes, in agreement with the conclusions formulated using Arias intensity as damage measure.

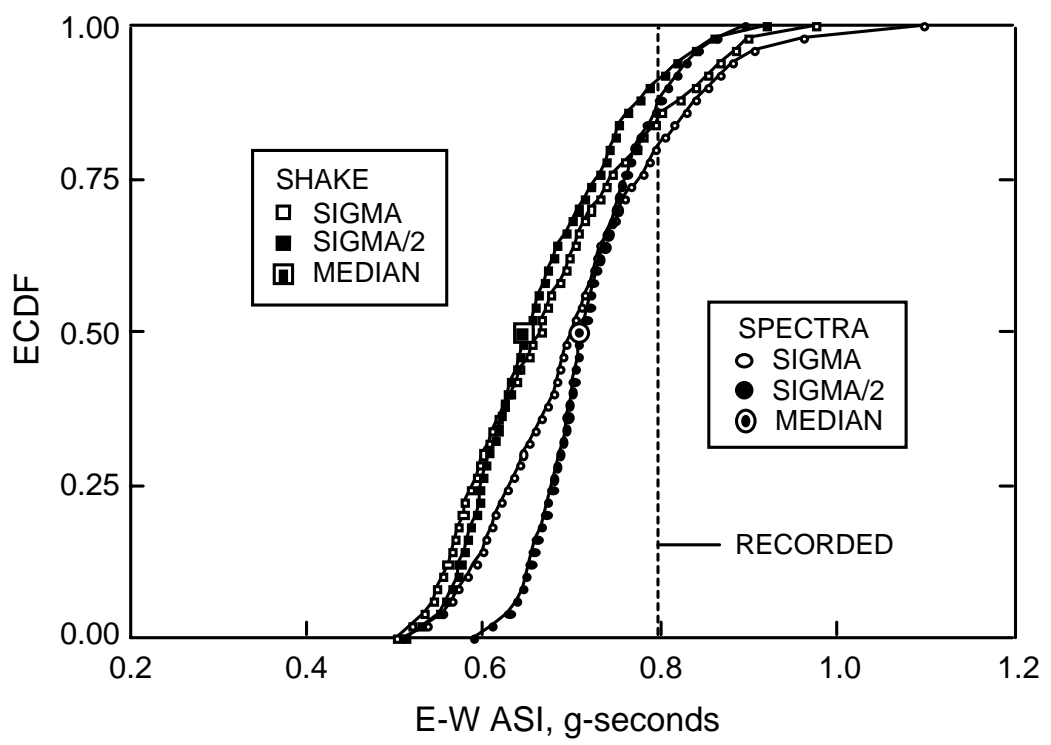

Fig. 10. ECDFs for Acceleration Spectrum Intensity (E-W direction) calculated at $T_{1}=20 \mathrm{~s}$ for LSST7 event using SHAKE and SPECTRA. Solid

ticks pertain to distributions obtained by using moduli reduction and damping curves with boundaries at two standard deviations, and empty ticks represent distributions obtained by reducing the original standard deviations by fifty percent.

\section{Summary and Conclusions}

We have performed combined stochastic-deterministic analyses of local site response using two computer codes, SHAKE and SPECTRA, to compare the sensitivities of equivalent linear and fully nonlinear analysis procedures to statistical variations in soil properties. The methodology consisted of propagating the uncertainties in soil properties using standard Monte Carlo simulations, utilizing the bedrock motion as a deterministic forcing function and choosing Arias intensity of the surface ground motion as the resulting response function. Sensitivities have been quantified in terms of the ECDFs of the response function. Three earthquakes having varied amplitudes, frequency contents, and duration, have been studied on soil deposits having varied geotechnical characteristics. Results of the sensitivity studies strongly suggest that SPECTRA exhibited about the same sensitivity as SHAKE. However, using median values of soil properties SPECTRA exhibited superior accuracy, predicting responses that were consistently closer to the recorded values compared to SHAKE. In terms of CPU costs, SPECTRA required more computer time with its more intensive calculations; however, 
we have performed and completed the sensitivity analyses on this code on a standard personal computer in a matter of a few hours. This suggests that it is possible to routinely quantify the sensitivity of this fully nonlinear algorithm for other response functions of interest, while concurrently enjoying the benefits of superior accuracy.

\section{Acknowledgments}

This work has been supported in part by National Science Foundation, Grant No. CMS0201317-001. The authors are grateful to: Prof. Charles Menun of Stanford University for his assistance with the structural reliability code CARDINAL; to Prof. C. Allin Cornell of Stanford University for providing manuscript copies of his papers; to three anonymous ASCE reviewers for their very constructive reviews; and to Dr. H.T. Tang and Electric Power Research Institute for making the digitized data for the Lotung site available. The first author acknowledges a Shah Family Research Assistantship through the Department of Civil and Environmental Engineering at Stanford University.

\section{References}

Anderson, D.G., and Tang, Y.K. (1989). "Summary of soil characterization program for the Lotung large-scale seismic experiment.” Proc. EPRI/NRC/TPC Workshop on Seismic Soil-Structure Interaction Analysis Techniques Using Data from Lotung, Taiwan, EPRI NP-6154, Electric Power Research Institute, Palo Alto Calif., vol 1, pp. 4.1-4.20.

Andrade, J.E., Menun C., and Borja, R.I. (2003). "Combined stochastic-deterministic analysis of local site response: A sensitivity study.” In: D. Doolin, A. Kammerer, T. Nogami, R.B. Seed, and I. Towhata (Eds.), Proc. 11th Int. Conf. Soil Dyn. Earthquake Eng., and 3rd Int. Conf. Earthquake Geotech. Eng., vol. 2, Stallion Press, pp. 76-81.

Arias, A. (1970). “A measure of earthquake intensity.” In: R.J. Hansen (Ed.), Seismic Design for Nuclear Power Plants, MIT, Cambridge, Mass., 438-483.

Baturay, M.B., and Stewart, J.P. (2003). "Uncertainty and bias in ground-motion estimates from ground response analyses.” Bull. Seism. Soc. Amer., 93(5), 20252042.

Bazzurro, P., and Cornell, C.A. (2004a). "Ground motion amplification in nonlinear soil sites with uncertain properties.” Bull. Seism. Soc. Amer., in press.

Bazzurro, P., and Cornell, C.A. (2004b). "Nonlinear soil site effects in probabilistic seismic hazard analysis.” Bull. Seism. Soc. Amer., in press. 
Berger, E., Fierz, H., and Kluge, D. (1989). "Predictive response computations for vibration tests and earthquake of May 20, 1986 using an axisymmetric finite element formulation based on the complex response method and comparison with measurements-a Swiss contribution," Proc. EPRI/NRC/TPC Workshop on Seismic Soil-Structure Interaction Analysis Techniques Using Data from Lotung, Taiwan, EPRI NP-6154, Electric Power Research Institute, Palo Alto Calif., vol 2, pp. 15.115.47 .

Borja, R.I., and Amies A.P. (1994). "Multiaxial cyclic plasticity model for clays.” J. Geotech. Eng., 120(6), 1051-1070.

Borja, R.I., Chao H.Y., Montáns F.J., and Lin C.H. (1999a). "Nonlinear ground response at Lotung LSST site.” J. Geotech. Eng., 125(3), 187-197.

Borja, R.I., Chao H.Y., Montáns F.J., and Lin C.H. (1999b). "SSI effects on ground motion at Lotung LSST site.” J. Geotech. Eng., 125(9), 760-770.

Borja, R.I., Lin, C.H., Sama, K.S., and Masada, G.M. (2000). "Modeling non-linear ground response of non-liquefiable soils," Earthquake Eng. Struct. Dyn., 29(1), 6383.

Borja R.I., Duvernay, B.G., and Lin, C.H. (2002). "Ground response in Lotung: Total stress analyses and parametric studies.” J. Geotech. Geoenviron. Eng., 128(1), 54-63.

Borja, R.I., Andrade, J.E., and Armstrong, R.J. (2004). "Combined deterministicstochastic analysis of local site response." Proc. 13th World Conf. Earthquake Eng., Vancouver, B.C., in CD-ROM.

Electric Power Research Institute (1993). "Guidelines for determining design basis ground motions-Vol.1: Method and guidelines for estimating earthquake ground motion in North America,” Tech. Rep. No. TR-102293, Electric Power Research Institute, Palo Alto, Calif.

Elgamal, A.W., Zeghal, M., Tang, H.T., and Stepp, J.C. (1995). "Lotung downhole array. I: Evaluation of site dynamic properties,” J. Geotech. Eng., 121(4), 350-362.

Faccioli, E. (1976). "A stochastic approach to soil amplification.” Bull. Seism. Soc. Amer., 66(4), 1277-1291.

Hardin, B.O., and Drnevich V.P. (1972). "Shear modulus and damping in soils: design equations and curves." J. Soil Mech. Found. Div., Am. Soc. Civ. Eng., 98(7), 667692.

Hashash, Y.M.A., and Park, D. (2002). "Viscous damping formulation and high frequency motion in non-linear site response analysis.” Soil Dyn. Earthquake Engrg., 22(7), 611-624. 
Hilber, H.M., Hughes, T.J.R., and Taylor, R.L. (1977). "Improved numerical dissipation for time-integration algorithms in structural dynamics.” Earthquake Eng. Struct. Dyn., 5(3), 283-292.

Hwang, H.H.M., and Huo, J.R. (1994). “Generation of hazard consistent ground motion.” Soil Dyn. Earthquake Eng., 13(6), 377-386.

Idriss, I.M., and Seed, H.B. (1968). “Seismic response of horizontal soil layers,” J. Soil Mech. Found. Div., Am. Soc. Civ. Eng., 94(4), 1003-1031.

Idriss, I.M., and Sun, J.I. (1992). User's Manual for SHAKE91, Center for Geotechnical Modeling, Univ. of California, Davis, Calif.

Kramer, S.L. (1996). Geotechnical Earthquake Engineering, Prentice-Hall, Englewood Cliffs, New Jersey.

Lee, M.K.W, and Finn, W.D.L. (1991). DESRA-2C: Dynamic effective stress response analysis of soil deposits with energy transmitting boundary including assessment of liquefaction potential. The University of British Columbia, Faculty of Applied Science, Vancouver, B.C.

Li, C., Borja, R.I., and Regueiro, R.A. (2004). "Dynamics of porous media at finite strain.” Comput. Methods Appl. Mech. Engrg., 193(36-38), 3837-3870.

Li, X.S., Wang, Z.L, and Shen, C.K. (1992). SUMDES: A nonlinear procedure for response analysis of horizontally-layered sites subjected to multi-directional earthquake loading. Dept. Civ. Eng., Univ. of California, Davis, Calif.

Pyke, R.M. (1992). TESS: A computer program for nonlinear ground response analyses. TAGA Engineering Systems and Software, Lafayette, California.

Roblee, C.J., Silva, W.J., Toro, G.R., and Abrahamson, N. (1996). "Variability in sitespecific seismic ground-motion design predictions.” In C.D. Schackelford, P.P. Nelson, and M.J.S. Roth (Eds.), Uncertainty in the Geologic Environment: From Theory to Practice, ASCE Geotech. Special Publication 58, Vol. 2, 1113-1133.

Schnabel, P.B., Lysmer, J., and Seed, H.B. (1972). "SHAKE---A computer program for earthquake response analyses of horizontally layered sites.” Report No. EERC 72-12, Univ. of California, Berkeley.

Seed, H.B., and Idriss, I.M. (1970). "Soil moduli and damping factors for dynamic response analysis.” EERC Rep. 70-10, Univ. of California, Berkeley, Calif.

Tang, H.T. (1987). "Large-scale soil-structure interaction.” EPRI NP-5513-SR Spec. Rep., Electric Power Research Institute, Palo Alto, Calif. 
Tsai, C.-C.P. (2000). "Probabilistic seismic hazard analysis considering nonlinear site effect.” Bull. Seism. Soc. Amer., 90(1), 66-72.

Von Thun, J.L., Rochim, L.H., Scott, G.A., and Wilson, J.A. (1988). "Earthquake ground motions for design and analysis of dams.” In Earthquake Engineering and Soil Dynamics II - Recent Advance in Ground Motion Evaluation, Geotechnical Special Publication 20, ASCE, New York, pp. 463-481.

Zeghal, M., Elgamal, A.W., Tang, H.T., and Stepp, J.C. (1995). "Lotung downhole array. II: Evaluation of soil nonlinear properties.” J. Geotech. Eng., 121(4), 363-378. 